\title{
Diffusion tensor imaging for assessment of normally appearing white matter of the brain and spinal cord in cases of multiple sclerosis: a multi-parametric correlation in view of patient's clinical status
}

Mohamed ElSayed Kamel Abdulaziz ElSayed ${ }^{1 *}$, Mohamed Mohamed Badawy El-Toukhy², Ramy Edward Asaad ${ }^{2}$ and Omar Amin El-Serafy ${ }^{3}$

\begin{abstract}
Background: Conventional MRI provides important morphologic information regarding the brain and spinal cord involvement by demyelinating plaques. However, it is of almost no value in assessing the normal-appearing white matter which has been proved by multiple pathologic studies to be directly and indirectly involved in the process of multiple sclerosis. Diffusion-weighted imaging and diffusion tensor imaging MRI have been widely used in multiple researches as a better solution for studying the normal-appearing white matter. The purpose of this study was to evaluate the role of diffusion tensor imaging examination of the normal-appearing white matter of the brain and spinal cord in patients with multiple sclerosis and to determine the relationship between diffusion tensor imaging metrics and patient's clinical status.

Results: The significant negative correlation found between expanded disability status scale score of secondary progressive MS patients and global (brain and spinal cord) average fractional anisotropy values in normal-appearing white matter and tracts $(P=0.000)$. Correlation between average apparent diffusion coefficient of corpus callosum tract and expanded disability status scale score revealed a significant positive correlation in RRMS $(P=0.001)$. While in secondary progressive MS, a significant negative correlation between fractional anisotropy average of the corpus callosum tract and expanded disability status scale score was noted $(P=0.015)$.

Conclusion: There is a strong relationship between diffusion tensor imaging readings and clinical status of patients can be used to understand unexplained deterioration over disease course and also can be used when conventional MRI findings are equivocal. Corpus callosum affection in MS patients is intimately related to clinical status and its assessment should be done whenever possible.
\end{abstract}

Keywords: Diffusion tensor magnetic resonance imaging, Fractional anisotropy, Apparent diffusion coefficient, Tractography, Multiple sclerosis, Normally appearing white matter

\footnotetext{
*Correspondence: dr_m_hendawy@msn.com

'Diagnostic and Interventional Radiology department, Police Authority

Hospitals, Ministry of Interior, Cairo, Egypt

Full list of author information is available at the end of the article
} 


\section{Background}

Multiple sclerosis (MS) is a chronic inflammatory/demyelinating and neurodegenerative disease of the central nervous system; in addition, it is the most common leading cause of non-traumatic disability in young and middle-age individuals. MS is estimated to affect more than 400,000 people in the USA and about 2 million worldwide. In Egypt, a study was conducted in 2008 showed that MS cases were $1.4 \%$ of all neurological diseases. The approximate total figure of patients in Egypt is around 50,000 [1].

Despite its increasing role in clinical management and scientific investigation of MS, conventional magnetic resonance imaging (MRI) is limited by having low pathological specificity and low sensitivity to diffuse damage in normal-appearing white matter (NAWM). In addition, conventional MRI shows a limited association with clinical status [2].

Diffusion tensor imaging (DTI) found to be more sensitive in identifying the occult tissue damage in MS patients than conventional magnetic resonance (MR) by means of accurate data about water diffusion within tissues. The DTI by its quantitative indices fractional anisotropy and mean diffusivity allows evaluation of the more widespread tissue damage occurring outside the lesions seen by conventional MRI [3].

DTI indices are considered non-invasive MR methods for studying the tissue structure. Fractional anisotropy (FA) measures the directionality of diffusion inside tissues while mean diffusivity (MD) is responsible for the estimation of mean diffusivity of water molecules within each voxel [4].

Diffusion abnormalities in NAWM have been shown in all types of MS, with micro-structural changes detected in early stages, even in early-onset MS. Furthermore, diffusion parameters differ according to the MS phenotype and within different lesions. Since MS subtypes demonstrate different diffusivity patterns, which may result from specific pathological substrates, diffusion indices may represent useful markers of different MS types. In addition, when diffusion measures are combined with other MR findings, they may give complementary information on different types of pathological damage caused by multiple sclerosis [5].

Lastly, given their high sensitivity in depicting structural tissue abnormalities, DTI measures have been identified as prognostic markers of disease course and as a means of monitoring anatomical changes over time [5].

\section{Methods}

\section{Aim of work}

The aim of this study was to evaluate the role of DTI MR examination of NAWM of the brain and spinal cord in patients with multiple sclerosis.
Furthermore, we also studied the DTI metrics in relapsing-remitting and secondary progressive MS subtypes in correlation with the clinical status of the patients in each MS group separately in a trial to statistically display the difference between both groups using DTI indices and expanded disability status scale (EDSS) scoring system.

\section{Patients}

The prospective randomized cross-sectional study comprised 50 right-handed ( 15 males and 35 females) patients who were referred from the Neurology Department after being clinically, laboratory, and radiologically diagnosed with multiple sclerosis (25 patients relapsing-remitting multiple sclerosis "RRMS" and 25 with secondary progressive multiple sclerosis "SPMS").

Twenty-five (14 males and 11 females) age-matched normal individuals were taken as control group.

Each patient included in the study was subjected to written informed consent before participation, full history taking, and examination, measuring EDSS clinical score (done by the neurologist).

Inclusion criteria for diagnosis of MS patients: I-RRMS; clinical criteria: two clear attacks typical of MS, documented by objective evidence in the central nervous system of two lesions separated in time and necessarily separated in space. Radiological criteria: MRI criteria of dissemination in space and time according to McDonald criteria. Exclusion of other disorders mimicking MS: usually by laboratory investigations (e.g., ischemia, systemic lupus erythematosus, Behçet syndrome, other vasculitides, HTLV-I, sarcoidosis). II-SPMS; initial RRMS disease course followed by gradual worsening with or without occasional relapses, minor remissions, and plateaus. At least 5 years after disease onset.

Exclusion criteria: patients with any absolute contraindications for MRI examination, e.g., cardiac pacemaker, intra-ocular foreign bodies. Age above 60 years.

\section{MRI examination}

The MR imaging was performed using a 1.5- $\mathrm{T}$ scanner (Ingenia, Philips) using Head Neck 20 channel coil. Patient position: supine.

Conventional MR examination including T1WI in axial plane, T2WI in axial, sagittal and coronal planes, and FLAIR in axial and sagittal planes. T1WI: TR 450, TE 15, matrix $80 \times 81$, FOV $230 \times 177$, slice thickness $5 \mathrm{~mm}$. T2WI: TR 3612, TE 100, matrix $208 \times 127$, FOV $230 \times$ 177, slice thickness $5 \mathrm{~mm}$. FLAIR: TR 6000, TE 120, matrix $240 \times 111$, FOV $230 \times 184$, slice thickness $5 \mathrm{~mm}$.

\section{Diffusion tensor imaging}

The DTI data were obtained using a single-shot echoplanar imaging sequence $(\mathrm{TR}=10.4 \mathrm{~s} / \mathrm{TE}=100 \mathrm{~ms})$ in 25 encoding directions. Diffusion gradient: $40 \mathrm{mT} / \mathrm{m}$, using a $b$ value of $800 \mathrm{~s} / \mathrm{mm}^{2}$. A FOV $=256 \times 256 \mathrm{~mm}^{2}$ 
and a data matrix $=128 \times 128$ were used. Image resolution $=2 \times 2 \times 2 \mathrm{~mm}^{3}$, number of slices $=30$, with a thickness of $2 \mathrm{~mm}$, with no gap, and with the total scan time $=10-12 \mathrm{~min}$.

\section{Post-processing image analysis}

DTI data were transferred to the workstation and assessed using (extended MR Workspace, Philips Medical Systems) software supplied by the manufacturer. The directionally encoded color FA and fused FLAIR/DTI maps were obtained. The region of interest (ROI) was placed in the normally appearing white matter while the affected white matter was excluded from measurements. FA and ADC indices were measured in each ROI (Fig. 1 and 2).

Application of multiple ROI in NAWM of the brain (ROI size 4-7 voxels) at bilateral frontal, bilateral high parietal (centrum semiovale, above level of lateral ventricles), bilateral low parietal (corona radiata, paraventricular location), bilateral temporal, bilateral occipital, and bilateral middle cerebellar peduncles as well as midbrain, pons and corpus callosum (genu, body and splenium).

Cervical cord (ROI size 7-10 voxels) opposite $\mathrm{C} 2-3$ and C6-7 disc levels as well as dorsal cord (ROI size 3-6 voxels) opposite D3-4 and D7-8 disc levels, spinal cord NAWM was also assessed.

Tractography of the corpus callosum and both corticospinal was performed and analyzed using the same parameters (FA and ADC) (Figs. 3 and 4).

\section{Statistical analysis}

The collected data was analyzed using Microsoft Office Excel 2003 and SPSS program (Statistical Package for Social Sciences) for Windows Version 20. Quantitative data were presented as mean \pm standard deviation (SD). For quantitative data, comparison between groups was done by $t$ test for independent samples. For qualitative data, relation between groups was done using the Pearson correlation. A $P$ value of 0.05 was chosen as a level of significance.

\section{Results}

In RRMS group, a comparison between the FA average in NAWM of brain and cord with that of the control group revealed significant decrease of FA average in spinal cord $(P=0.033)$ while decrease of the FA average of the NAWM of the brain was insignificant $(P=0.445)$.

When the same comparison was done in the SPMS group, a significant decrease in FA average of NAWM of

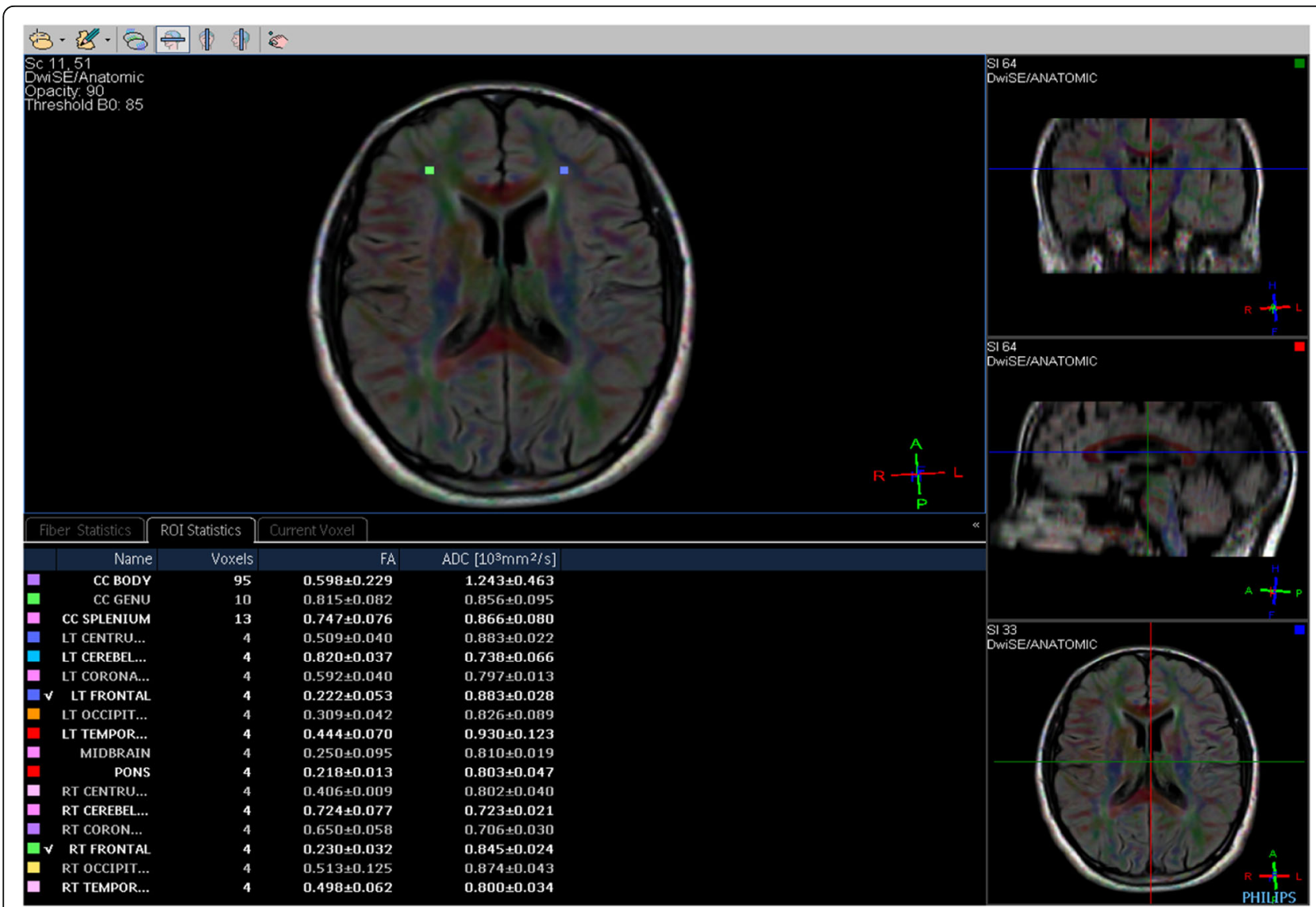

Fig. 1 ROI-based FA and ADC measurement in the brain 


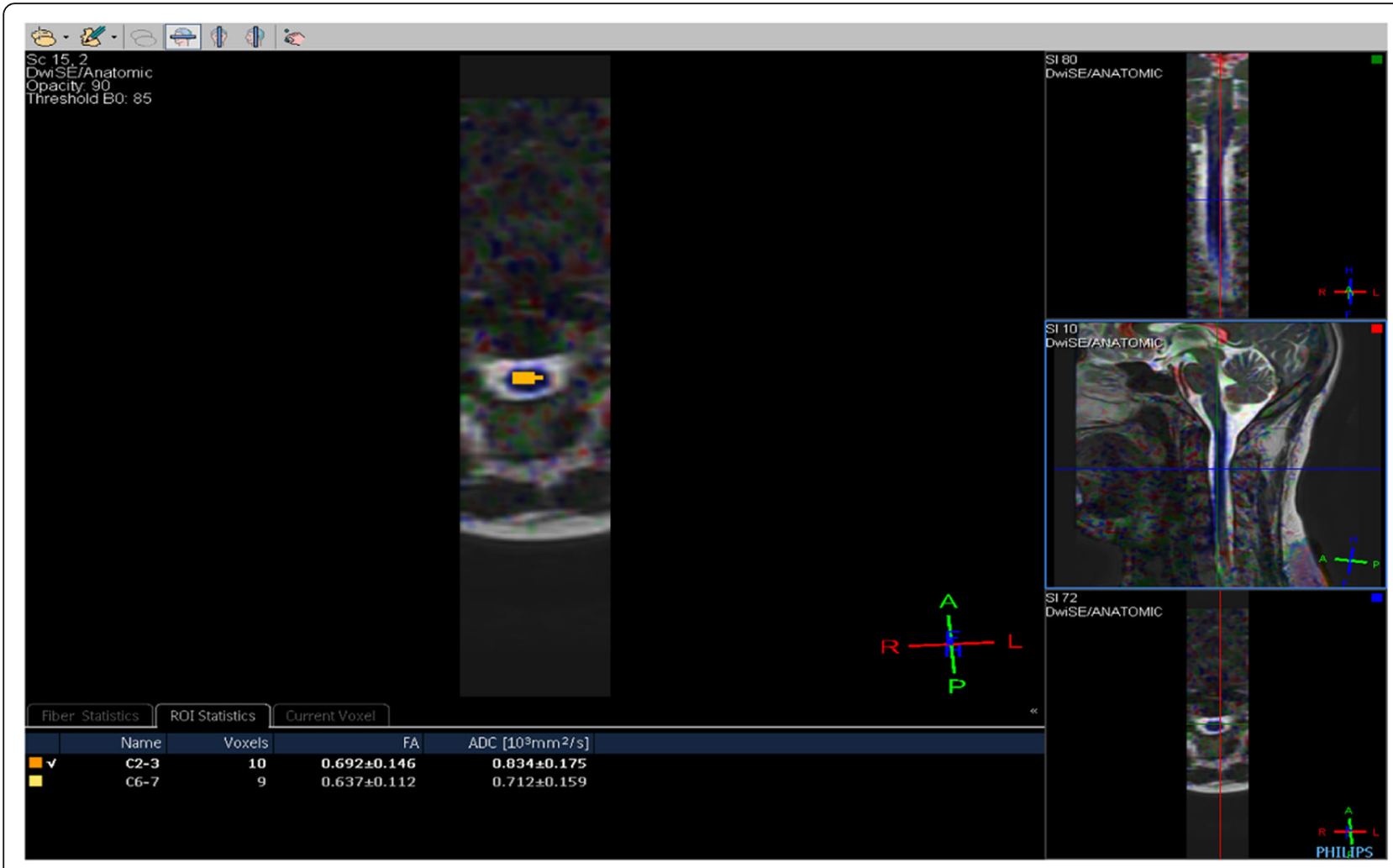

Fig. 2 ROI-based FA and ADC measurement in the spinal cord



Fig. 3 Tractography of the corpus callosum 
combined (global) brain and spinal cord was found compared to the control group $(P=0.007)$, and regarding FA average of the brain and spinal cord separately, the decrease of FA in spinal cord $(P=0.006)$ was more significant than that noted in the brain $(P=0.038)$.

Similarly, the comparison using the apparent diffusion coefficient (ADC) values in NAWM of the brain and spinal cord of both patient groups (comparing each group with the control group individually) and the $t$ test results were as follows:

- I-RRMS group:

- Global ADC average significantly increased $(P=$ $0.000)$.

- Brain ADC average significantly increased $(P=$ 0.030).

- Spinal cord ADC average significantly increased $(P=0.000)$.

- II-SPMS group:

- Global ADC average significantly increased $(P=$ $0.000)$.

- Brain ADC average significantly increased $(P=$ 0.000).

- Spinal cord ADC average significantly increased $(P=0.000)$.

The increase in ADC average was more significant in SPMS group than in relapsing RRMS group and in the spinal cord more than the brain regions.

Similarly, a comparison was done between average ADC values for corpus callosum and both cortico-spinal tracts in control and both study groups. In RRMS group, a significant increase in ADC values for corpus callosum tract only $(P=0.009)$ was noted. In SPMS group, the ADC average increased significantly for corpus callosum $(P=0.000)$, left $(P=0.000)$ and right $(P=0.000)$ cortico-spinal tracts.

A comparison between the FA and ADC measures in different regions was conducted, comparing the RRMS group and SPMS group to the normal control group and using the $t$ test in order to measure the $P$ value to determine which region displayed statistically significant changes. Regions that showed combined significant decrease of FA and increase of ADC in both groups were left frontal, right frontal, pons, and dorsal cord at D7-8 level (Tables 1 and 2).

Table $1 P$ value of FA decrease per region

\begin{tabular}{lll}
\hline Region & $\begin{array}{l}\text { RRMS compared } \\
\text { to control }\end{array}$ & $\begin{array}{l}\text { SPMS compared } \\
\text { to control }\end{array}$ \\
\hline LT frontal & $0.000^{* *}$ & $0.005468^{* *}$ \\
Pons & $0.0145^{*}$ & $0.000^{* *}$ \\
RT frontal & $0.000^{* *}$ & $0.01774^{*}$ \\
D7-8 & $0.049953^{*}$ & $0.049395^{*}$ \\
\hline
\end{tabular}

* Significant at $P<0.05$

**Highly significant at $P<0.01$
Table $2 P$ value of ADC increase per region

\begin{tabular}{lll}
\hline Region & $\begin{array}{l}\text { RRMS compared } \\
\text { to control }\end{array}$ & $\begin{array}{l}\text { SPMS compared } \\
\text { to control }\end{array}$ \\
\hline LT frontal & $0.001707^{* *}$ & $0.000962^{* *}$ \\
Pons & $0.00012^{* *}$ & $0.006378^{* *}$ \\
RT frontal & $0.012494^{*}$ & $0.000^{* *}$ \\
D7-8 & $0.000^{* *}$ & $0.000^{* *}$ \\
\hline
\end{tabular}

*Significant at $P<0.05$

${ }^{*}$ Highly significant at $P<0.01$

Correlation between DTI findings and clinical score (EDSS) In SPMS group, the EDSS was negatively correlated with the global average FA in NAWM and tracts (Pearson correlation $=0.765)(P=0.000)($ Table 3$)$.

When doing a correlation between the previously mentioned regions that showed combined significant decrease of FA and increase of ADC values (left frontal, right frontal, pons, and dorsal cord as D7-8 level) and the EDSS in the two MS subtypes, RRMS group showed only significant correlation with the ADC of right frontal region and it was a positive correlation (Pearson correlation $=0.547$ ) $(P=0.005)$, similar findings were noted in SPMS group showing statistically significant positive correlation with EDSS (Pearson correlation $=0.417)(P=0.038)$

On the other hand, when using FA parameter, negative correlations were noted in SPMS group between EDSS score and FA values of the right frontal (Pearson correlation $=0.428)(P=0.033)$ and pons (Pearson correlation $=0.601)(P=0.001)$ regions (Table 4$)$.

In RRMS group, a highly significant positive correlation was noted between average ADC of corpus callosum tract and the EDSS (Pearson correlation $=0.63)(P=0.001)$, whereas in SPMS group, significant negative correlation was noted between average FA of corpus callosum tract and the EDSS (Pearson correlation $=0.482)(P=0.015)$ (Table 5).

\section{Discussion}

DTI takes the advantage of diffusion of water in brain tissue within three main directions, which is decreased perpendicularly to the myelin sheaths and cell membranes of white-matter axons [6].

In the present study, the RRMS group showed a significant reduction of NAWM FA in the spinal cord only with a significant increase of NAWM ADC in both brain and spinal cord. In SPMS, the FA reduction was significant in both brain and spinal cord but more significant in the spinal cord than in the brain and as regards the ADC increase, it was significant in the brain as well as the spinal cord.

Concerning the spinal cord, the present study agreed with previous DTI studies of the spinal cord in MS patients reported a statistically significant FA difference between the control group and the MS patient group [3, 7-11]. 


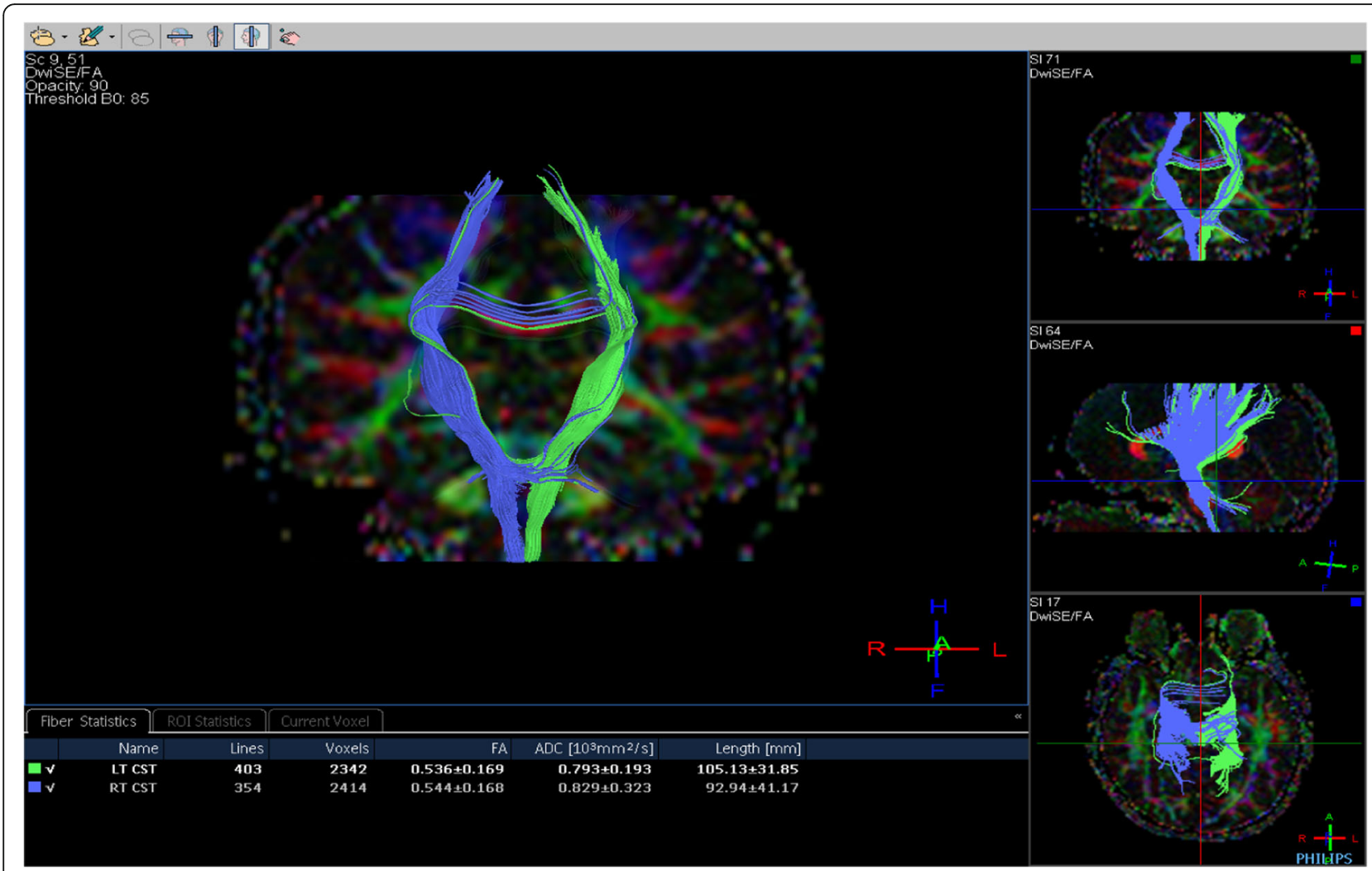

Fig. 4 Tractography of both cortico-spinal tracts

Chen et al. [7] stated that the decrease in the FA value of NAWM and the increase in MD value as compared to normal controls indicate the presence of occult damage to the NAWM.

Fatehi et al. [8] concluded that MD values in NAWM of the patients were higher than NAWM of healthy individuals. The FA values also were lower in NAWM of the patients compared to NAWM of the healthy individuals.

Gratsias et al. [9] showed that NAWM is indeed affected in the patients with MS, regardless of its identification on conventional MR images. The FA and ADC values of NAWM were decreased and increased respectively in comparison with the FA and ADC values of the symmetrical white matter (WM) of the control group.

The present study results also suggest that the spinal cord is not preserved in MS even if lesions are only detected in the brain and MS cord pathology seems to be independent of concomitant brain changes as well as to develop at different rates according to disease subtype.
Bergers et al. [12] confirmed extensive axonal loss in postmortem analysis of the spinal cords of patients with MS, largely independent of local lesion load seen at T2weighted MR imaging.

Moreover, the present results showed that RRMS patients have milder structural affection of NAWM (especially in the brain) than SPMS patients as indicated by the discrepancy of FA reduction in the brain being significant in SPMS patients only.

Cercignani et al. [13] and Nusbaum et al. [14] stated that DTI changes are significantly more severe in patients with SPMS than in those with RRMS supporting a role for DTI in monitoring advanced stages of the disease.

In addition, FA affection of the NAWM of the spinal cord of both groups with only brain affection in SPMS group, could be explained by the fact that spinal cord has a small surface area compared to the brain, so RRMS patients with milder structural white matter changes among large surface area of the brain could escape detection by

Table 3 Correlation between average FA in NAWM and tracts and EDSS in secondary progressive group

\begin{tabular}{lllll}
\hline & Mean & Std. deviation & Pearson correlation & Significance \\
\hline FA in NAWM and tracts & 0.5379 & 0.03555 & 0.765 & $0.000^{* *}$ \\
EDSS & 5.6400 & 0.79739 & & \\
\hline
\end{tabular}

**Correlation is highly significant at $P<0.01$ 
Table 4 Correlation between ADC/FA per region and EDSS in both MS groups

\begin{tabular}{llllll}
\hline & & Mean & Std. deviation & Person correlation & Significance \\
\hline RR MS & EDSS & 2.1000 & 0.87797 & & 0.547 \\
SP MS & ADC of right frontal & 0.8334 & 0.05849 & & $0.005^{* *}$ \\
& EDSS & 5.6400 & 0.79739 & 0.417 & $0.038^{*}$ \\
& ADC of right frontal & 0.8782 & 0.06872 & 0.428 & $0.033^{*}$ \\
& FA of right frontal & 0.3727 & 0.12261 & 0.601 & $0.001^{* *}$ \\
& FA of pons & 0.3355 & 0.10231 & &
\end{tabular}

*Correlation is significant at $P<0.05$

${ }^{* *}$ Correlation is highly significant at $P<0.01$

DTI at the time that similar changes in small surface area of spinal cord are easily detectable by DTI. Hence, DTI sensitivity is affected by the surface area of the studied tissues and the smaller the surface area, the more sensitive the DTI measures.

The present study showed that ADC affection appears to occur in an earlier stage than FA values do, as indicated by significant ADC increase in RRMS patients' brain with still no significant FA changes. This coincides with considering $\mathrm{ADC}$ as an indicator of demyelination and FA for axonal degeneration.

Werring and colleagues also found that a steady and moderate elevation of ADC values can precede the development of new plaques. Such finding suggests that new lesions are preceded by progressive, albeit subtle, tissue abnormalities beyond the resolution of conventional MRI [15].

We could explain these results as follows: MD parameter is primarily influenced by free space hence its increase with vasogenic edema, inflammation as well as myelin loss which occurs first in the disease process while FA is more sensitive to the detection of the integrity of WM which is affected later on.

However, Agosta et al. [16] stated that astrocytic proliferation, cell debris, fibrillary gliosis, and inflammatory infiltrates can result in a (pseudo) normalization of the $M D$ values and can, therefore, prevent the MD differences to be statistically significant. This can explain the presence of some normal or even low ADC values in our study.

Considering DTI affection of brain tracts, the present study revealed significant ADC changes; in
RRMS, there was a significant ADC increase in corpus callosum only, while in SPMS the ADC values increased significantly in the corpus callosum and both cortico-spinal tracts. The FA results were insignificant.

Temel et al. [11] showed that mean fiber ADC value was statistically significantly higher than that in the control group.

In the present study, the regions that showed the most significant decrease in FA and increase in ADC in NAWM of the brain and spinal cord in both study disease groups compared to the normal control group were as follows:

In RRMS group, left frontal, right frontal, pons, and dorsal cord at D7-8 level. So we can say that in spite of the insignificant decrease of global average FA in NAWM of the brain in the relapsing-remitting group, there were certain regions within the brain that showed significant FA decrease namely both frontal regions and pons.

Meanwhile, in SPMS group, the most significant FA and ADC changes were noted at the following regions: left frontal, right frontal, pons, dorsal cord at D7-8 level, corpus callosum splenium, right occipital, and cervical cord at C2-3 level.

Once again, this confirms that white matter affection in SPMS patients is more advanced than in those with RRMS.

Consequently, in the present study, the left frontal, right frontal, pons, and dorsal cord at D7-8 level regions were the most significantly affected areas of the NAWM in the brain and spinal cord respectively (in both RRMS and SPMS groups) regarding the changes in DTI parametric FA and ADC indices.

Table 5 Correlation between average ADC/FA of corpus callosum tract and EDSS in both MS groups

\begin{tabular}{|c|c|c|c|c|c|}
\hline & & Mean & Std. deviation & Pearson correlation & Significance \\
\hline \multirow[t]{2}{*}{ RRMS } & EDSS & 2.1000 & 0.87797 & & \\
\hline & $\mathrm{ADC}$ of $\mathrm{CC}$ tract & 0.9173 & 0.04863 & 0.63 & $0.001^{* *}$ \\
\hline \multirow[t]{2}{*}{ SP MS } & EDSS & 5.6400 & 0.79739 & & \\
\hline & FA of CC tract & 0.4222 & 0.04507 & 0.482 & $0.015^{*}$ \\
\hline
\end{tabular}

${ }^{*}$ Correlation is significant at $P<0.05$

${ }^{* *}$ Correlation is highly significant at $P<0.01$ 
Karami et al. [17] demonstrated that the values in the bilateral frontal peri-ventricular WM of the brain were much higher than the other parts.

The present study yielded no significant correlation between FA and ADC indices and the clinical score (EDSS) in RRMS group. This may be attributed to the short disease duration, less severity, and mild white matter affection in those patients together with the fact that most of them suffer from mild motor disability with low EDSS scores.

Patients of SPMS group experienced longer disease duration, more aggressive and extensive white matter affection with higher EDSS scores. This was the cause of the strong highly significant negative correlation found in our study between the EDSS score and global (brain, spinal cord, and white matter tracts) average FA values. This means that the more FA reduction the more EDSS score increase.

Filippi et al. [18] found a mild negative and positive correlation between NAWM mean FA and mean ADC values, respectively, and the EDSS score of patients with
SPMS, whereas no correlation was noted with EDSS score in RRMS patients.

Karami et al. [17] have found that there is a significant correlation between DTI indices and EDSS scores in MS patients. One of the reasons was the higher EDSS scores of this study (EDSS $=2.6$ ) compared with other studies EDSS scores (EDSS < 2.6). Fatehi et al. [8] also found similar results.

The present study disagreed with $\mathrm{Yu}$ et al. [19], Temel et al. [11], and Rovira and Leon [20] where they found no significant correlation between diffusion parameters and EDSS scores. This may be attributed to different statistical software in DTI analysis, small sample size, or presence of mild clinical disability in their patients.

Based on that, if a multiple sclerosis patient with high EDSS score and the conventional MRI findings do not match with this score, as well as his heavy motor disability cannot be explained by conventional imaging, we can subject this patient for further assessment by DTI MR so his symptoms can be explained; in addition, we can

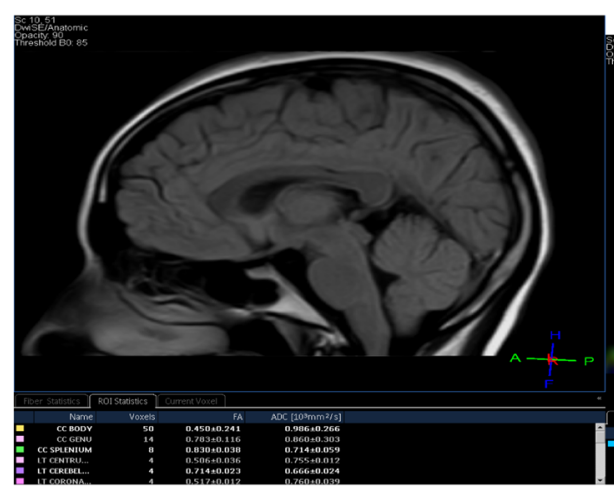

a

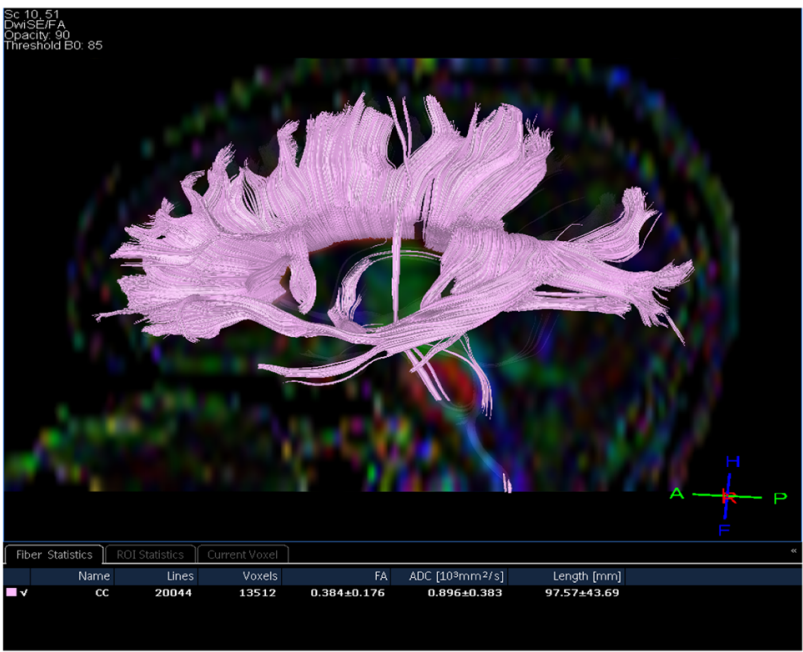

c

\section{b}

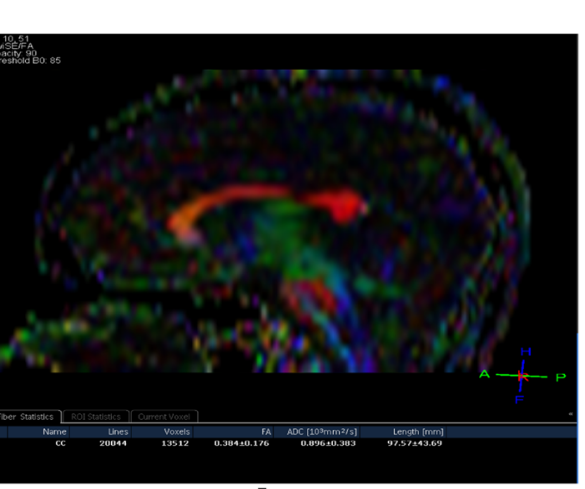

Fig. 5 Patient with RRMS. a Morphological apparently normal conventional FLAIR sagittal image of the corpus callosum. b Directionally encoded tractography color map of the same sagittal section of the corpus callosum shows a small defect in the posterior part of its body. c Tractography of the corpus callosum confirms defective fibers at the same location 
expect that this patient is classified as having secondary progressive phenotype or at least is going to progress to the secondary progressive MS eventually. These DTI results can be a justification to give this patient some medication that is used for treatment or prevention from progression to SPMS.

Considering the previously mentioned regions that displayed the most significant FA and ADC changes (both frontal, pons, and dorsal cord at D7-8 level), our study results revealed that in RRMS the only significant correlation was a positive one between the ADC of the right frontal region and the EDSS score. The same was noted in the SPMS group as well.

In RRMS group, the FA/region did not show any significant correlation with EDSS score at any of the above-mentioned regions, this was in line with our result regarding the non-significant FA reduction in NAWM of the brain in those patients and the fact that axonal degeneration is not widely seen among those patients.

On the other hand, in SPMS the FA/region, values were significant negatively correlated with the EDSS score in two regions namely the right frontal region and pons.

Based on the present study results, it seems that the right frontal region is the most significantly affected region by the disease process in both groups as confirmed by the changes in the non-conventional magnetic resonance DTI indices and moreover the changes in this region were most significantly correlated with the patient's clinical status and motor disability in both RRMS and SPMS groups.

Considering the present study results regarding the corpus callosum tract (being the most morphologically affected tract), these supported the forementioned results that showed more ADC increase in RRMS patients due to demyelination and more FA reduction in SPMS group due to axonal degeneration. This also reflects that affection of the corpus callosum in MS patient is intimately related to the clinical status of patients (Fig. 5).

Sigal et al. [21] focusing on the corpus callosum, concluded that changes in ADC and specifically in transverse diffusivity represent microstructural damage in RRMS and positively correlated with clinical activity and progression of the disease.

Rimkus et al. [22] results showed a correlation between mean diffusion and radial diffusivity, and extended disability status scale (EDSS), suggesting a possible relationship between demyelination of corpus callosum and the motor/sensory dysfunction.

Caligiuri et al. [23] conducted a study which revealed that EDSS score correlated with regions of the corpus callosum bundle crossed by motor and pre-motor fibers.

In both studied MS subgroups, DTI provides insight on the pathology of NAWM that can help to differentiate relapsing-remitting from secondary progressive MS subtypes, better understand the clinical correlates, and help in monitoring the evolution of the disease process.

DTI can be also performed in cases with clinically suspected spinal cord plaques where conventional MRI lacks sensitivity.

Future modifications of dissemination in time (DIT) and dissemination in space (DIS) McDonald criteria taking DT-MRI changes into considerations is also a matter of time and further studies for longitudinal follow-up is highly indicated for fully establishing the role of DTI examination of the NAWM in diagnosis and management of patients with multiple sclerosis.

\section{Conclusion}

There is considerable affection of NAWM detected in MS patients beyond the visible disease load in conventional images with the predilection of SPMS phenotype.

There is a strong relationship between DTI readings and the clinical status of the patient in the form of EDSS score that can be used to understand the unexplained deterioration over the disease course and also can be used when conventional MRI findings are equivocal.

Corpus callosum affection in MS patients is intimately related to the clinical status and its assessment (qualitative and quantitative) should be done whenever possible in all MS patients.

\section{Abbreviations}

ADC: Apparent diffusion coefficient; CC: Corpus callosum; CST: Cortico-spinal tract; DIS: Dissemination in space; DIT: Dissemination in time; DTI: Diffusion tensor imaging; DWI: Diffusion-weighted imaging; EDSS: Expanded Disability Status Scale; FA: Fractional anisotropy; MD: Mean diffusivity; MS: Multiple sclerosis; NAWM: Normal-appearing white matter; ROl: Regions of interest; RRMS: Relapsing-remitting multiple sclerosis; SPMS: Secondary-progressive multiple sclerosis

\section{Acknowledgements}

Special thanks to Amr Samir Wahdan, lecturer of anesthesia and pain management at Faculty of Medicine in Cairo University for his great effort in participation in the statistical analysis of this work.

\section{thics approval and consent to participate}

This study was approved by the Research Ethics Committee of the Faculty of Medicine at Cairo University in Egypt on 13 July 2015; reference number of approval: 228062002. All patients included in this study gave written informed consent to participate in this research. If the patient was less than 16 years old or unconscious at the time of the study, written informed consent for their participation was given by their parent or legal guardian.

\section{Authors' contributions}

MMBE and OAE gave the idea. REA put study design. MEKAE collected the patients' data and analyzed them as well as wrote the paper with revision. They all approved the final version of the manuscript.

\section{Funding}

This study had no funding from any resource.

Availability of data and materials

The datasets used and/or analyzed during the current study are available from the corresponding author on reasonable request. 


\section{Consent for publication}

All patients included in this research gave written informed consent to publish the data contained within this study. If the patient was less than 16 years old, deceased, or unconscious when consent for publication was requested, written informed consent for the publication of this data was given by their parent or legal guardian.

\section{Competing interests}

The authors declare that they have no competing interests.

\section{Author details}

'Diagnostic and Interventional Radiology department, Police Authority Hospitals, Ministry of Interior, Cairo, Egypt. 'Diagnostic and Interventional Radiology department, Faculty of Medicine, Cairo University, Cairo, Egypt. ${ }^{3}$ Neurology department, Faculty of Medicine, Cairo University, Cairo, Egypt.

Received: 1 August 2019 Accepted: 5 August 2019

Published online: 06 September 2019

\section{References}

1. Egyptian Society of Neurology, Psychiatry, and Neurosurgery (ESNPN) (2013), the MS Chapter, Cairo.

2. Lassmann H, Bruck W, Lucchinetti C (2001) Heterogeneity of multiple sclerosis pathogenesis: implications for diagnosis and therapy. Trends Mol Med. 7(3):115-121

3. Testaverde L, Caporali L, Venditti E, Grillea G, Colonnese C (2012) Diffusion tensor imaging applications in multiple sclerosis patients using 3T magnetic resonance: a preliminary study. Eur Radiol 22:990-997

4. Razek AAKA, El-Serougy L, Abdelsalam M, Gaballa G, Talaat M (2018) Differentiation of residual/recurrent gliomas from postradiation necrosis with arterial spin labeling and diffusion tensor magnetic resonance imaging-derived metrics. Neuroradiology 60:169-177

5. Sbardella E, Tona F, Petsas N, and Pantano P. (2013) DTI Measurements in multiple sclerosis: evaluation of brain damage and clinical implications, Multiple Sclerosis International, Article ID 671730, 11 pages, https:/doi.org/10.1155/2013/671730.

6. El-Serougy L, Abdel Razek AA, Ezzat A, Eldawoody H, El-Morsy A (2016) Assessment of diffusion tensor imaging metrics in differentiating low-grade from high-grade gliomas. Neuroradiol J 29:400-407

7. Chen J, Zhou C, Zhu L, Yan X, Wang Y, Chen X And Fang S. (2017) Magnetic resonance diffusion tensor imaging for occult lesion detection in multiple sclerosis. Exp Ther Med, 13: 91-96.

8. Fatehi D, Naleini F, Salehi MG, Gharib A, Farzizadeh M, Rostamzadeh A. (2016) Evaluation of multiple sclerosis patients through structural biomarkers of diffusion tensor magnetic imaging and correlation with clinical features. J Chem Pharm Sci. JCPS, Volume 9 Issue 2.

9. Gratsias G, Kapsalaki E, Kogia S, Dardiotis E, Tsimourtou V, Lavdas E, Kousi E, Pelekanou A, Hadjigeorgiou GM, Fezoulidis I (2015) A quantitative evaluation of damage in normal appearing white matter in patients with multiple sclerosis using diffusion tensor MR imaging at 3 T. Acta Neurol Belg 115:111-116

10. Elshafey R, Hassanien O, Khalil M (2014) Diffusion tensor imaging for characterizing white matter changes in multiple sclerosis. Egypt J Radiol Nucl Med 45:881-888

11. Temel \$̧, Kekliğkoğlu HD, Vural G, Deniz O, Ercan K (2013) Diffusion tensor magnetic resonance imaging in patients with multiple sclerosis and its relationship with disability. Eur Neurol J 26:3-17

12. Bergers E, Bot JC, De Groot CJ, Polman CH, a` L, Nijeholt GJ, Castelijns JA et al (2002) Axonal damage in the spinal cord of MS patients occurs largely independent of T2 MRI lesions. Neurology 59:1766-1771

13. Cercignani M, Bozzali M, lannucci G (2001) Magnetisation transfer ratio and mean diffusivity of normal appearing white and grey matter from patients with multiple sclerosis. J Neurol Neurosurg Psychiatry 70(3):311-317

14. Nusbaum AO, Tang CY, Wei T (2000) Whole-brain diffusion MR histograms differ between MS subtypes. Neurology. 54(7):1421-1427

15. Werring DJ, Brassat D, Droogan AG et al (2007) The pathogenesis of lesions and normal-appearing white matter changes in multiple sclerosis. A serial diffusion MRI study. Brain 123(8):1667-1676

16. Agosta F, Absinta M, et al. (2007) In vivo assessment of cervical cord damage in MS patients: a longitudinal diffusion tensor MRI study. Brain 130 (Pt 8):2211-2219.
17. Karami V, Mahdavifar R, Nabavi SM (2016) Association assessment between diffusion tensor magnetic resonance imaging indices and clinical disabilities in ms patients. Biomedical Engineering: Applications, Basis and Communications 28(05):1650034. https://doi.org/10.4015/S1 016237216500344

18. Filippi M, Cercignani M, Inglese $M$ et al (2001) Diffusion tensor magnetic resonance imaging in multiple sclerosis. Neurology 56:304-311

19. Yu CS, Lin FC, Liu Y, Duan Y, Lei H, Li KC (2008) Histogram Analysis Of Diffusion Measures In Clinically Isolated Syndromes And RelapsingRemitting Multiple Sclerosis. Euro J Radiol 68:328-334

20. Rovira A, Leon A (2008) MR in the Diagnosis And Monitoring Of Multiple Sclerosis: An Overview. Euro J Radiols 67:409-414

21. Sigal T, Shmuel M, Mark D, Gil H, Anat A (2012) Diffusion tensor imaging of corpus callosum integrity in multiple sclerosis: correlation with disease variables. J Neuroimaging 22:33-37

22. Rimkus CM, Junqueira TF, Lyra KP, Jackowski MP, Machado MA, Miotto EC, Callegaro D, Otaduy MC, Leite CC (2011) Corpus callosum microstructural changes correlate with cognitive dysfunction in early stages of relapsingremitting multiple sclerosis: axial and radial diffusivities approach. Mult Scler Int 304875

23. Caligiuri ME, Barone $S$, Cherubini A, Augimeri A, Chiriaco C, Trotta M, Granata A, Filippelli E, Perrotta P, Valentino P, Quattrone A (2015) The relationship between regional microstructural abnormalities of the corpus callosum and physical and cognitive disability in relapsing-remitting multiple sclerosis. Neurolmage: Clinical 7:28-33

\section{Publisher's Note}

Springer Nature remains neutral with regard to jurisdictional claims in published maps and institutional affiliations.

\section{Submit your manuscript to a SpringerOpen ${ }^{\circ}$ journal and benefit from:}

- Convenient online submission

- Rigorous peer review

- Open access: articles freely available online

High visibility within the field

- Retaining the copyright to your article

Submit your next manuscript at $\boldsymbol{\nabla}$ springeropen.com 